PSICOLOGIA

IBEROAMERICANA
Psicología Iberoamericana ISSN: 1405-0943

revista.psicologia@ibero.mx

Universidad Iberoamericana, Ciudad de México México

\title{
Síndrome de Boreout en trabajadores baby boomers, $x$ y millennials de la Ciudad de México
}

García-González, Bryan E; Villavicencio-Ayub, Erika; García, Melissa

Síndrome de Boreout en trabajadores baby boomers, $x$ y millennials de la Ciudad de México

Psicología Iberoamericana, vol. 26, núm. 2, 2018

Universidad Iberoamericana, Ciudad de México, México

Disponible en: http://www.redalyc.org/articulo.oa?id=133959841010 


\section{Síndrome de Boreout en trabajadores baby boomers, x y millennials de la Ciudad de México}

\section{BOREOUT SYNDROME IN BABY BOOMER, X AND MILLENNIAL EMPLOYEES FROM MÉXICO CITY}

Bryan E García-González

Universidad Nacional Autónoma de México, México

bryan.garciagonzalez@yahoo.com

Erika Villavicencio-Ayub

Universidad Nacional Autónoma de México, México

Melissa García

Universidad Nacional Autónoma de México, México
Redalyc: http://www.redalyc.org/articulo.oa? $\mathrm{id}=133959841010$

Recepción: 23 Marzo 2018

Aprobación: 05 Marzo 2019

\section{Resumen:}

Se evaluaron los niveles del síndrome Boreout en trabajadores mexicanos, utilizando la Escala de Síndrome de Boreout, adaptada para la población mexicana (Barrios \& Velasco, 2016). Se realizó un estudio no experimental, de corte trasversal. Participaron 268 trabajadores con edades entre 19 y 70 años, de las generaciones baby boomer, x y millennial, de los cuales el $54 \%$ fueron hombres. Se esperaba que el síndrome se presentara en niveles altos en millennials, en niveles moderados en la generación $\mathrm{x}$ y en niveles bajos en baby boomers. No obstante, se encontró que los niveles del síndrome de Boreout fueron generalmente bajos y no difirieron entre generaciones $(F(2,265)=2.597, \mathrm{p}>.05)$. Se discute sobre la necesidad de seguir desarrollando métodos para evaluar el síndrome de boreout con la finalidad de diseñar intervenciones efectivas para su tratamiento y medidas preventivas para el mismo.

PALABRAS ClaVE: Síndrome de Boreout, infraexigencia laboral, baby boomer, generación x, millennial.

\section{Abstract:}

Boreout Syndrome levels were evaluated in Mexican employees, using the Boreout Syndrome Scale adapted for the Mexican population (Barrios \& Velasco, 2016). A non-experimental, cross-sectional study was performed. 268 employees between the ages of 19 and 70 years old participated. Participants were from Baby Boomer, X and Millennial generations and 54\% were men. It was expected that the Syndrome was higher in Millennials, spread moderately in X Generation and low in Baby Boomers. Instead, it was found that Boreout Syndrome levels were generally low and did not differ among generations $(F(2,265)=2.597, p>.05)$. The need to continue developing methods for evaluating the Boreout Syndrome is discussed in this article, with the aim to design effective interventions for its treatment and preventive measures for it.

KeYWORDs: Boreout Syndrome, job infraexigency, Baby Boomer, X Generation, Millennial .

\section{INTRODUCCIÓN}

En las últimas décadas, el interés por el estudio de los factores psicosociales en el mundo laboral ha incrementado debido a que han aparecido nuevas formas de organización y con ello nuevos problemas laborales (Cabrera Noriega, 2014). Algunos de estos problemas involucran disminuciones en el rendimiento de los trabajadores ocasionadas en gran medida por las condiciones laborales a las que son expuestos (Peiró \& Tetrick, 2011). Dentro de estos casos se encuentra el síndrome de Boreout o síndrome de aburrimiento laboral, el cual se ha descrito como una situación de aburrimiento crónico que resulta en un estado de desinterés por el trabajo y por la organización, debida a los bajos niveles de exigencia laboral (Rothlin \& Werder, 2009). Las personas diagnosticadas con síndrome de aburrimiento laboral reportan sentir falta de motivación, pérdida de la concentración, cansancio al llegar a su lugar de trabajo, apatía hacia cualquier actividad laboral y un sentimiento de frustración constante debido a que sus habilidades superan las 
requeridas para realizar las tareas que les son asignadas (Aguilar-Pérez \& Tapia-García, 2013). De manera paralela a esta situación de malestar, los trabajadores comienzan a realizar actividades diferentes a las del trabajo como navegar en internet, hablar por teléfono y chatear, con la finalidad de relajarse. Sin embargo, la situación se sale de control y se convierte en una distracción que les impide cumplir con sus funciones laborales (Gómez-Tenorio \& Tapia-García, 2013). Para disimular su bajo rendimiento laboral y evitar que les sean asignadas nuevas tareas, los trabajadores aparentan estar ocupados en el trabajo, emitiendo conductas como transportar papeles de un lugar a otro, simular mantener llamadas telefónicas de trabajo, realizar comentarios acerca de su excesiva carga laboral y fingir llevar trabajo a casa (Barrios \& Velasco, 2016).

El síndrome de aburrimiento laboral disminuye la productividad de las organizaciones, por lo que resulta en una serie de costos económicos. De hecho, en algunas empresas estadounidenses se ha reportado que los costos monetarios debidos a los bajos niveles de productividad asociados al síndrome fueron mayores a los 750 millones de dólares al año, lo que fue equivalente a más de 5 mil dólares por trabajador al año (Cabrera Noriega, 2014; Rothlin \& Werder, 2009). Debido a los altos costos que representa el síndrome de aburrimiento laboral para las organizaciones, se han comenzado a investigar los factores de riesgo asociados al síndrome. No obstante, la ausencia de instrumentos de medición ha dificultado su evaluación, por lo que la literatura a nivel internacional es escasa y actualmente no se cuenta con datos para México.

Algunos de los factores de riesgo asociados al síndrome de aburrimiento laboral que se han descrito en la literatura, incluyen que las funciones del trabajador estén por debajo de sus capacidades (i. e., infraexigencia laboral), que las actividades que realiza sean monótonas, tediosas y carezcan de un sentido personal y que no exista la autonomía en la organización, respecto a la toma de decisiones (Cabrera Noriega, 2014). Adicionalmente, se ha mencionado que este síndrome aparece cuando el esfuerzo de los trabajadores no es valorado adecuadamente a nivel económico, ni a nivel de reconocimiento social, así como por la imposibilidad de promocionarse y ascender en su línea de carrera (Azabache, 2016).

En la literatura se ha descrito que, además de las condiciones laborales a las que son expuestos los trabajadores, algunas características personales pueden ser un factor de riesgo para el desarrollo de algunas enfermedades ocupacionales. Una de ellas es la generación a la que pertenecen los trabajadores. De manera general, una generación se refiere a un grupo de personas que nació en un periodo determinado bajo condiciones políticas, económicas y sociales particulares que han influido de manera sistemática en el comportamiento de dichas personas (Strauss \& Howe, 1997). Al respecto, se ha reportado que la adicción al trabajo se presenta con mayor frecuencia en la generación de baby boomers en comparación con trabajadores de la generación x y de los millennials (Gibson, Greenwood, \& Murphy, 2009). Esta diferencia se ha atribuido a que la generación de baby boomers tiende a ser competitiva y a dedicar una cantidad excesiva de tiempo a su trabajo, de tal manera que descuidan aspectos de su vida personal, mientras que los $\mathrm{x}$ y los millennials tienden a mantener un equilibrio entre su vida laboral y su vida personal (Crampton \& Hodge, 2006; Lawrence, 2005). Respecto a los millennials, se ha reportado que tienden a aburrirse en el trabajo con mayor facilidad que las personas pertenecientes a las generaciones x y baby boomers (Gibson et al., 2009). Esto último es importante porque plantea la posibilidad de que, así como la adicción al trabajo tiende a presentarse principalmente en baby boomers, el síndrome de aburrimiento laboral pueda presentarse principalmente en millennials. No obstante, hasta la fecha se desconoce si el síndrome se presenta diferencialmente en trabajadores de distintas generaciones.

Las condiciones laborales actuales a las que son expuestos los trabajadores mexicanos parecen contar con algunos factores de riesgo asociados al síndrome de aburrimiento laboral como son la monotonía de las tareas, la falta de ascensos de puesto, los salarios bajos y el bajo reconocimiento social, por lo que parece posible que este síndrome se presente en múltiples escenarios. Debido a los altos costos económicos y humanos en los que resulta el síndrome para las organizaciones, el interés del presente estudio fue evaluar los niveles de síndrome de aburrimiento laboral en trabajadores mexicanos de las tres generaciones que predominan en el campo laboral, utilizando la Escala de Síndrome de Boreout adaptada para población mexicana (Barrios \& Velasco, 
2016). La hipótesis fue que el síndrome se presentaría en niveles altos en millennials, en niveles moderados en la generación $\mathrm{x}$ y en niveles bajos en baby boomers.

\section{MÉTODO}

\section{Diseño}

Con el objetivo de comparar los niveles del síndrome de aburrimiento laboral entre trabajadores mexicanos de diferentes generaciones, se realizó un estudio no experimental, de corte transversal.

\section{Participantes}

Participaron 268 trabajadores de organizaciones públicas y privadas de la Ciudad de México. Los participantes fueron seleccionados por conveniencia, considerando como requisitos ser mexicanos, estar laborando actualmente y tener más de 18 años. Se contó con la participación de 31 baby boomers, 108 personas de la generación x y 129 millennials. Se consideró como baby boomers a aquellos nacidos entre 1943 y 1960 , a los x como aquellos nacidos entre 1961 y 1981, y a los millennials como aquellos nacidos después de 1982. Los rangos de edad fueron tomados de Strauss y Howe (1997). Las características sociodemográficas de los participantes se resumen en la Tabla 1.

TABLA 1

Características sociodemográficas de los trabajadores de cada generación

\begin{tabular}{|c|c|c|c|c|c|}
\hline Generación & Sexo & Escolaridad & Sector laboral & Estado civil & Puesto \\
\hline Baby boomers & $\begin{array}{l}\text { Hombres } 67.7 \% \\
\text { Mujeres } 32.3 \%\end{array}$ & $\begin{array}{l}\text { Primaria } 9.7 \% \\
\text { Secundaria } 29 \% \\
\text { Bachillerato } 25.8 \% \\
\text { Licenciatura } 25.8 \% \\
\text { Posgrado } 9.7 \%\end{array}$ & $\begin{array}{l}\text { Privado } 58.1 \% \\
\text { Público } 41.9 \%\end{array}$ & $\begin{array}{l}\text { Soltero } 16.1 \% \\
\text { Casado } 74.2 \% \\
\text { Otro } \\
9.7 \%\end{array}$ & $\begin{array}{l}\text { Operativo } 61.3 \% \\
\text { Medio } 29 \% \\
\text { Directivo } 9.7 \%\end{array}$ \\
\hline Generación x & $\begin{array}{l}\text { Hombres } 48.1 \% \\
\text { Mujeres } 51.9 \%\end{array}$ & $\begin{array}{l}\text { Primaria } 13 \% \\
\text { Secundaria } 25.9 \% \\
\text { Bachillerato } 28.7 \% \\
\text { Licenciatura } 26.9 \% \\
\text { Posgrado } 5.9 \%\end{array}$ & $\begin{array}{l}\text { Privado } 76.9 \% \\
\text { Público } 23.1 \%\end{array}$ & $\begin{array}{l}\text { Soltero } 21.3 \% \\
\text { Casado } 61.1 \% \\
\text { Otro } \\
17.6 \%\end{array}$ & $\begin{array}{l}\text { Operativo } 78.7 \% \\
\text { Medio } 17.6 \% \\
\text { Directivo } 3.7 \%\end{array}$ \\
\hline Millennials & $\begin{array}{l}\text { Hombres } 55.8 \% \\
\text { Mujeres } 44.2 \%\end{array}$ & $\begin{array}{l}\text { Primaria } 3.1 \% \\
\text { Secundaria } 27.9 \% \\
\text { Bachillerato } 31 \% \\
\text { Licenciatura } 35.7 \% \\
\text { Posgrado } 2.3 \%\end{array}$ & $\begin{array}{l}\text { Privado } 80.6 \% \\
\text { Público } 19.4 \%\end{array}$ & $\begin{array}{l}\text { Soltero } 62 \% \\
\text { Casado } 17.8 \% \\
\text { Otro } 20.2 \%\end{array}$ & $\begin{array}{l}\text { Operativo } 74.4 \% \\
\text { Medio } 24 \% \\
\text { Directivo } 1.6 \%\end{array}$ \\
\hline
\end{tabular}

\section{Instrumentos}

Se utilizó la Escala de Síndrome de Boreout adaptada para población mexicana de Barrios y Velasco (2016), adaptada de la escala original de Cabrera Noriega (2014). El instrumento cuenta con un alfa de Cronbach de .81 y está conformado por tres factores distribuidos en 14 reactivos. Los factores que conforman el 
instrumento son: desinterés laboral (tres reactivos), infraexigencia laboral (cuatro reactivos) y sobrecarga de trabajo imaginaria (siete reactivos). Algunos de los reactivos que conforman los factores son "prefiero platicar con mis compañeros que hacer mi trabajo" para desinterés laboral, "puedo dar más de lo que el jefe me exige" para infraexigencia laboral y "cuando salgo del trabajo procuro cargar documentos para aparentar que tengo mucho trabajo" para sobrecarga de trabajo imaginaria. Los reactivos cuentan con cuatro opciones de respuesta en una escala tipo Likert que va desde nunca, casi nunca, casi siempre y siempre (ver Anexo 1).

\section{Procedimiento}

La escala se aplicó durante el periodo de junio a septiembre de 2017 de manera manual utilizando lápiz y papel o virtualmente mediante la plataforma de inventarios de Google. En ambos casos, se presentó un consentimiento informado en el cual se le explicó a los trabajadores que su participación era totalmente voluntaria, que se mantendría el anonimato de la información recabada y que ésta sería utilizada exclusivamente con fines de investigación. Finalmente, se agradeció su participación y se les proporcionó información sobre la investigación. Las instrucciones utilizadas fueron las siguientes:

La Facultad de Psicología de la unam está realizando una investigación sobre el aburrimiento laboral que presentan los trabajadores mexicanos. A continuación, se presenta un conjunto de afirmaciones. Por favor seleccione la opción de respuesta que mejor describa su caso. Cabe aclarar que no existen respuestas correctas o incorrectas. Sus respuestas serán anónimas y se utilizarán únicamente con fines de investigación. Se solicita que únicamente responda el cuestionario si usted se encuentra laborando actualmente.

\section{Resultados}

Se compararon los niveles del síndrome de aburrimiento laboral en trabajadores mexicanos de diferentes generaciones a través de los puntajes obtenidos en Escala de Síndrome de Boreout (Barrios \& Velasco, 2016). De acuerdo con los valores del instrumento, una puntuación global dentro de un rango de cero a 13 se consideró como un nivel mínimo del síndrome, con valores de 14 a 26 un nivel bajo, con valores de 27 a 40 un nivel medio y con valores de 41 a 56 un nivel alto. En la Figura 1 se muestra el porcentaje de trabajadores que presentaron el síndrome de aburrimiento laboral según sus diferentes niveles de manera global y para cada una de las generaciones. 

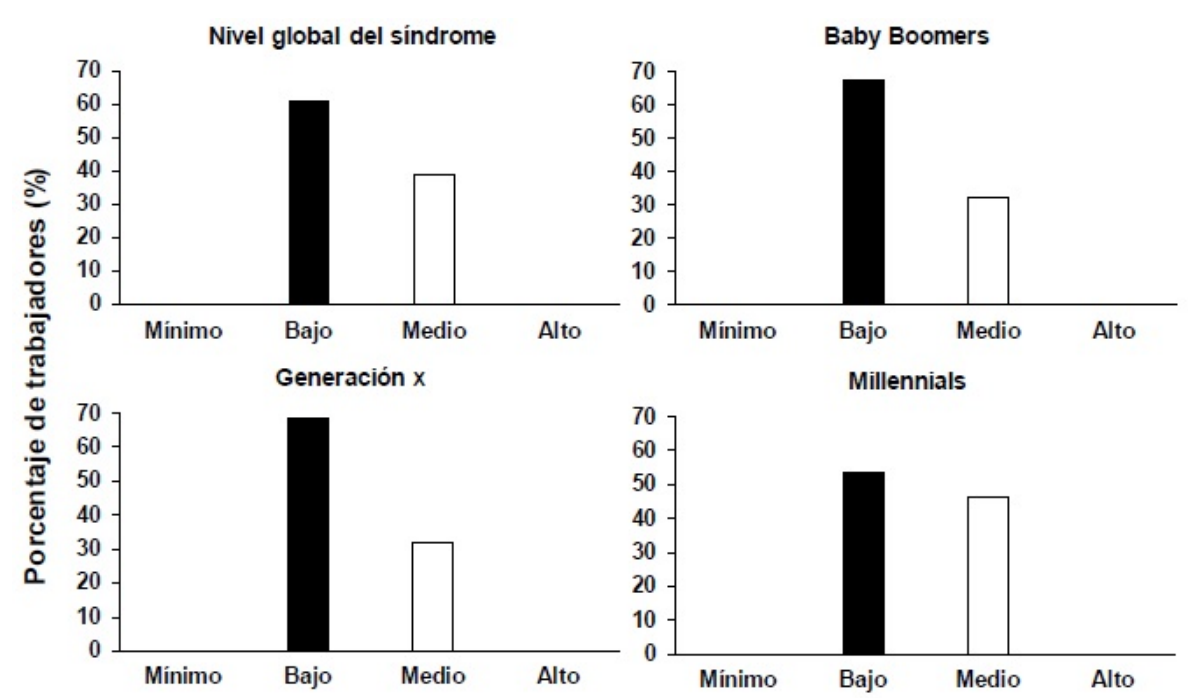

FIGURA 1

Nivel del síndorme de aburrimiento laboral

Porcentaje de trabajadores que presentaron el síndrome de aburrimiento laboral según sus diferentes niveles: mínimo, bajo, medio y alto. En el panel superior izquierdo se muestra el porcentaje total de los trabajadores, en el panel superior derecho se muestra el porcentaje de los trabajadores baby boomers, en el panel inferior izquierdo se muestra el porcentaje de los trabajadores de la generación $\mathrm{x}$ y en el panel inferior derecho se muestra el porcentaje de los trabajadores millennials.

En la presente investigación, el síndrome de aburrimiento laboral se presentó únicamente en niveles bajos y medios, siendo el nivel bajo el que predominó en las tres generaciones ( $=25.49, \mathrm{DE}=3.57)$. Se encontró que las puntuaciones globales fueron similares entre trabajadores baby boomers $(=24.80, \mathrm{DE}=3.71$ ), “ $\mathrm{x}$ " ( $=25.18, \mathrm{DE}=3.46)$ y millennials $(=26.06, \mathrm{DE}=3.57)$. Asimismo, se encontró que los puntajes obtenidos en los tres factores del instrumento fueron similares para los trabajadores de cada generación (ver Tabla 2).

TABLA 2

Medias y desviaciones estándar de los puntajes obtenidos en los tres factores de la escala de síndrome de boreout en trabajadores de distintas generaciones

\begin{tabular}{|c|c|c|c|}
\hline Generación & $\begin{array}{l}\text { Desinterés } \\
\text { laboral }\end{array}$ & $\begin{array}{c}\text { Infraexigencia } \\
\text { laboral }\end{array}$ & $\begin{array}{c}\text { Sobrecarga } \\
\text { de trabajo } \\
\text { imaginaria }\end{array}$ \\
\hline $\begin{array}{c}\text { Baby } \\
\text { boomers }\end{array}$ & $\begin{array}{c}=2.10 \\
(\mathrm{DE}=.58)\end{array}$ & $\begin{array}{c}=1.98 \\
(\mathrm{DE}=.33)\end{array}$ & $\begin{array}{c}=1.31 \\
(\mathrm{DE}=.34)\end{array}$ \\
\hline $\begin{array}{c}\text { Generación } \\
x\end{array}$ & $\begin{array}{c}=2.11 \\
(\mathrm{DE}=.49)\end{array}$ & $\begin{array}{c}=1.95 \\
(\mathrm{DE}=.28)\end{array}$ & $\begin{array}{c}=1.30 \\
(\mathrm{DE}=.36)\end{array}$ \\
\hline Millennials & $\begin{array}{c}=2.09 \\
(D E=.44)\end{array}$ & $\begin{array}{c}=2.02 \\
(\mathrm{DE}=.30)\end{array}$ & $\begin{array}{c}=1.41 \\
(\mathrm{DE}=.38)\end{array}$ \\
\hline
\end{tabular}

Para determinar si las diferencias en las puntuaciones globales fueron estadísticamente significativas, según la generación a la que pertenecían los trabajadores, se realizó un anova de una vía. De acuerdo con este análisis, no se encontraron diferencias estadísticamente significativas entre las puntuaciones globales (F (2, $265)=2.597, \mathrm{p}>.05)$. De manera similar, se realizó un anova de una vía para comparar las puntuaciones 
obtenidas en cada factor del instrumento. De acuerdo con los análisis, no se encontraron diferencias estadísticamente significativas entre las puntuaciones obtenidas en desinterés laboral $(\mathrm{F}(2,265)=.060, \mathrm{p}>.05)$, infraexigencia laboral $(\mathrm{F}(2,265)=2.888, \mathrm{p}>.05)$ y sobrecarga de trabajo imaginaria $(\mathrm{F}(2,265)=2.815$, $\mathrm{p}>.05$ ). Adicionalmente se analizaron las puntuaciones globales y las obtenidas en cada uno de los tres factores del instrumento, según las diferentes variables sociodemográficas utilizando las pruebas $t$ de Student para muestras independientes y anova de una vía, según el caso. No se encontraron diferencias estadísticamente significativas al comparar por sexo, estado civil, sector laboral o puesto. Las únicas diferencias que se encontraron fueron por escolaridad, tanto en las puntuaciones globales $(\mathrm{F}(4,263)=6.415, \mathrm{p}<.05)$ como en las obtenidas en desinterés laboral $(\mathrm{F}(4,263)=4.789, \mathrm{p}<.05)$, infraexigencia laboral $(\mathrm{F}(4,263)=2.724$, $\mathrm{p}<.05)$ y sobrecarga de trabajo imaginaria $(\mathrm{F}(4,263)=3.536, \mathrm{p}<.05)$. Sin embargo, al analizar el tamaño del efecto de las diferencias, se encontró que, aunque fueron estadísticamente significativas, estas resultaron ser irrelevantes $(\eta 2=.089 ; \eta 2=.068 ; \eta 2=.040$ y $\eta 2=.051$, respectivamente).

\section{DISCUSIÓN}

Las condiciones laborales a las que son expuestos los trabajadores mexicanos cuentan con una gran cantidad de factores de riesgo asociados al síndrome de aburrimiento laboral. Al respecto, se esperaba que el síndrome se presentara en niveles altos en millennials, en niveles moderados en la generación $\mathrm{x}$ y en niveles bajos en baby boomers. No obstante, los participantes de la presente investigación reportaron bajos niveles del síndrome. Este hallazgo fue similar al reportado por Daza, Plaza y Hernández (2017), quienes encontraron que el $78 \%$ de los trabajadores colombianos que participaron en su estudio mostraron bajos niveles del síndrome, mientras que el $21 \%$ mostró niveles moderados y sólo el 1\% niveles altos. Aunque aparentemente el síndrome de aburrimiento laboral se presenta en niveles bajos en trabajadores mexicanos, es importante considerar que sus respuestas pudieron sesgarse por deseabilidad social, al tratar de dar respuestas que consideraran apropiadas para los investigadores o para la organización en la que laboraban, por lo que existe la posibilidad de que la presencia del síndrome se haya subestimado en el presente estudio. Esta posibilidad, sugiere la incorporación de otros indicadores para evaluar el síndrome como son el cumplimiento de objetivos por parte de los trabajadores, el número de errores que se presentan durante la jornada laboral e incluso los posibles accidentes laborales ocasionados por la falta de concentración en sus actividades.

A pesar de que en la literatura se ha descrito que algunas enfermedades ocupacionales tienden a predominar en personas de generaciones específicas (e. g., la adicción al trabajo en Baby Boomers; Gibson et al., 2009), parece ser que el síndrome de aburrimiento laboral no se presenta diferencialmente entre baby boomers, x y millennials mexicanos. Este hecho sugiere que las variables que resultan en el síndrome se presentan de la misma manera en los trabajadores de las tres generaciones. Sin embargo, es importante destacar que, la presente investigación consistió en una primera aproximación al fenómeno, por lo que las conclusiones que se pueden obtener a partir de los datos son limitadas.

Debido a que la presente investigación fue de tipo exploratoria, es importante considerar detalles metodológicos relacionados con el instrumento utilizado, pues además de la deseabilidad social, una segunda variable que podría explicar los bajos niveles del síndrome en los participantes y que pudo oscurecer las diferencias entre los niveles de aburrimiento laboral de los trabajadores de las distintas generaciones es el estrecho rango de valores posibles en la escala de respuesta, ya que sólo estuvieron disponibles cuatro opciones (nunca, casi nunca, casi siempre y siempre) y al menos el $85 \%$ de las respuestas de los participantes se encontraron entre el primer y el segundo valor de la escala (nunca y casi nunca, respectivamente) en diez de los 14 reactivos del instrumento, de tal manera que la variabilidad entre las respuestas de los participantes se pudo ver limitada. En futuras investigaciones se podrían utilizar instrumentos psicométricos con una mayor cantidad de opciones de respuesta, con la finalidad de amplificar la variabilidad de las respuestas de los participantes. 
La ausencia de métodos de evaluación ha limitado la investigación sobre el síndrome de aburrimiento laboral, por lo que las investigaciones empíricas sobre el tema son escasas. Hasta donde los autores saben, esta investigación es el primer reporte empírico realizado con trabajadores mexicanos y es la primera comparación entre trabajadores de diferentes generaciones, al respecto del síndrome. Al tratarse de una aproximación inicial al fenómeno, el presente trabajo es susceptible de ser mejorado en diferentes aspectos. Algunos de los posibles cursos de acción consistirían en seleccionar participantes de diferentes entidades de la República de manera aleatoria, con el objetivo de generalizar los resultados a la población mexicana, así como equiparar el número de trabajadores de cada generación para realizar las comparaciones, pues en el presente estudio, la limitada disponibilidad de participantes resultó en que el número de participantes de cada generación fuera considerablemente diferente entre sí. Adicionalmente, los trabajadores de cada generación podrían ser equiparados según las distintas variables sociodemográficas, con el propósito de analizar sus efectos aisladamente al respecto del síndrome. Además, sería importante considerar que en algunas ocasiones las personas podrían cometer errores al momento de evaluarse, otorgándose una calificación más favorable en comparación con la evaluación que darían otras personas sobre la misma característica (i. e., error de indulgencia; Gil-Flores, 2007). Este hecho es importante, ya que al momento de utilizar autoreportes para evaluar el síndrome, dicho reporte podría sesgarse y resultar en una especie de autoindulgencia. En futuras investigaciones se podrían utilizar métodos de observación directa que permitan registrar algunas de las conductas relacionadas con el síndrome de aburrimiento laboral (e. g., chatear) en los escenarios naturales con la finalidad de cotejar los registros conductuales con las respuestas verbales recabadas con instrumentos psicométricos, así como con otros indicadores del síndrome como el cumplimiento de objetivos.

El síndrome de aburrimiento laboral constituye una condición desfavorable para los trabajadores y para las organizaciones, pues resulta en una sensación de malestar a nivel personal y en una pérdida de productividad para la organización. Por ello, es necesario realizar investigaciones que nos permitan identificar las variables que resultan en este síndrome, con la finalidad de imple-

mentar intervenciones efectivas para su tratamiento y desarrollar medidas preventivas para el mismo. Al respecto, la escasa evidencia empírica sugiere que proveer de autonomía a los trabajadores, establecer desafíos laborales y disponer de métodos innovadores y creativos en lugar de procedimientos estandarizados pueden reducir los efectos negativos del síndrome (Stock, 2015; 2016). Sin embargo, aún queda mucho por descubrir sobre los factores psicosociales relacionados con el síndrome y será de suma importancia adaptar las estrategias organizacionales a través de la diversidad que existe entre organizaciones, generaciones y culturas. En conclusión, el síndrome de aburrimiento laboral representa uno de los nuevos retos en salud ocupacional y requiere de investigaciones empíricas para el desarrollo de métodos para su detección y manejo oportuno.

\section{REFERENCIAS}

Aguilar-Pérez, V. M., \& Tapia-García, O. (2013). El síndrome de Boreout. Primer Congreso Estatal de Ciencias Administrativas, 1, 3-9.

Azabache, K. A. (2016). Construcción y propiedades psicométricas de la escala del síndrome de boreout - esb en colaboradores adultos de Trujillo. (Tesis doctoral). Universidad César Vallejo, Perú.

Barrios, C. R., \& Velasco, J. B. (2016). Adaptación de un instrumento para medir el sindrome de aburrimiento al trabajo (boreout) en población laboral mexicana. (Tesis de licenciatura no publicada). México: Universidad Nacional Autónoma de México.

Cabrera Noriega, L. E. (2014). Síndrome de Boreout: Diseño, confiabilidad y validación preliminar de un instrumento para su medición. Revista de la Universidad Industrial de Santander Salud, 46(3), 259-265.

Crampton, S. M., \& Hodge, J. W. (2006) The supervisor and generational differences. Academy of Organizational Culture, Communications and Conflict: Proceedings, 11(2), 19-22. 
Bryan E García-GonzÁlez, et aL. Síndrome de Boreout en trabajadores baby boomers, x y miLlenNials...

Daza, D., Plaza, M. T., \& Hernández, H. E. (2017). Factores de riesgo intralaboral asociados al clima organizacional y su efecto sobre el síndrome de boreout en el sector salud. Revista Espacios, 38(58), 3.

Gibson, J. W., Greenwood, R. A., \& Murphy, E. F. Jr. (2009). Generational differences in the workplace: Personal values, behaviors and popular beliefs. Journal of Diversity Management, 4(3), 1-8. doi:10.19030/jdm.v4i3.4959

Gil-Flores, J. (2007). La evaluación de competencias laborales. Educación XXI, 10, 83-106.

Gómez-Tenorio, E. D., \& Tapia- García, O. (2013). El síndrome del Boreout, sus estadios y estrategias. Primer Congreso Estatal de Ciencias Administrativas, 1, 52-58.

Lawrence, G. S. (2005). Medical professionalism and the generation gap. Association of Professors of Medicine, 118(4), 439-442. doi:10.1016/j.amjmed.2005.01.021

Peiró, J. M., \& Tetrick, L. (2011). Occupational health psychology. LAAP handbook of applied psychology. London, Reino Unido: Wiley-Blackwell.

Rothlin, P., \& Werder, P. (2009) Elnuevo sindrome laboral boreout, recupera la motivación. New York, NY: De Bolsillo.

Stock, R. M. (2015). Is boreout a threat to frontline employees' innovative work behavior? Journal of Product Innovation Management, 32(4), 574-592. doi:10.1111/jpim.12239

Stock, R. M. (2016). Understanding the relationship between frontline employee boreout and customer orientation. Journal of Business Research, 69(10), 4259-4268. doi:10.1016/j.jbusres.2016.02.037

Strauss, W., \& Howe, N. (1997). The fourth turning: What the cycles of history tell us about America's next rendezvous with destiny. New York, NY: Broadway Books. 


\section{ANEXO 1}

Escala de sindrome de boreout adaptada para población mexicana (Barrios \& Velasco, 2016)

\section{Datos sociodemográficos}

\begin{tabular}{|c|c|c|c|c|c|}
\hline Edad: & \multirow{2}{*}{\multicolumn{5}{|c|}{$\begin{array}{l}\text { Sexo: } \\
\text { Estado Civil: }\end{array}$}} \\
\hline Puesto u ocupación: & & & & & \\
\hline \multicolumn{6}{|l|}{ Escolaridad: } \\
\hline \multirow[t]{2}{*}{ Sector en el que trabaja: } & \multicolumn{2}{|l|}{ Público } & \multicolumn{2}{|c|}{ Privado } & \\
\hline & & Nunca & $\begin{array}{c}\text { Casi } \\
\text { nunca }\end{array}$ & $\begin{array}{c}\text { Casi } \\
\text { siempre }\end{array}$ & Siempre \\
\hline \multicolumn{2}{|l|}{ 1. Prefiero platicar con mis compañeros que hacer mi trabajo. } & & & & \\
\hline \multicolumn{2}{|c|}{ 2. La cantidad de trabajo que tengo va de acuerdo con mi capacidad. } & & & & \\
\hline \multicolumn{2}{|c|}{ 3. Solicito a mi jefe más tiempo del necesario para realizar un trabajo. } & & & & \\
\hline \multicolumn{2}{|c|}{$\begin{array}{l}\text { 4. Me preocupo en hacer con un nivel de excelencia mis actividades } \\
\text { en el trabajo. }\end{array}$} & & & & \\
\hline \multicolumn{2}{|l|}{ 5. Puedo dar más de lo que el jefe me exige. } & & & & \\
\hline \multicolumn{2}{|l|}{ 6. Hago pausas largas mientras realizo un proyecto. } & & & & \\
\hline \multicolumn{2}{|c|}{ 7. Si me atraso en un proyecto en equipo culpo a mis compañeros. } & & & & \\
\hline \multicolumn{2}{|c|}{$\begin{array}{l}\text { 8. Cuando salgo del trabajo procuro cargar documentos para aparentar } \\
\text { que tengo mucho trabajo. }\end{array}$} & & & & \\
\hline \multicolumn{2}{|c|}{$\begin{array}{l}\text { 9. Si puedo evadir por un tiempo la responsabilidad de una tarea laboral } \\
\text { me siento relajado. }\end{array}$} & & & & \\
\hline \multicolumn{2}{|c|}{$\begin{array}{l}\text { 10. Si estoy ocupado en actividades ajenas al trabajo trato de que nadie } \\
\text { lo note. }\end{array}$} & & & & \\
\hline \multicolumn{2}{|l|}{ 11. Puedo pasar horas sin saber qué hacer en el trabajo. } & & & & \\
\hline \multicolumn{2}{|l|}{ 12. En el trabajo ocupo tiempo para proyectos personales. } & & & & \\
\hline \multicolumn{2}{|c|}{$\begin{array}{l}\text { 13. Me quedo más tiempo en el trabajo, aunque ya no tenga nada } \\
\text { que hacer. }\end{array}$} & & & & \\
\hline 14. Dejo documentos sin revisar o correos por varios días. & & & & & \\
\hline
\end{tabular}

